\title{
Phase Sensitive Amplifiers for Regeneration of Phase Encoded Optical Signal Formats
}

\author{
Joseph Kakande*, Francesca Parmigiani, Radan Slavík, Periklis Petropoulos, and David J. Richardson \\ Optoelectronics Research Centre, University of Southampton, Southampton, SO17 1BJ, UK \\ Tel: +44 (0)238059 4521 , Fax: (0)23 8059 3142,e-mail: jkk@orc.soton.ac.uk
}

\begin{abstract}
We discuss the application of phase sensitive fiber optical parametric devices for the regenerative processing of high baud rate optical signals. We present recent advances in phase-sensitive amplification technology and its application to the regeneration of phase-encoded signals. By combining four wave mixing based parametric effects in highly nonlinear optical fibers and injection locking assisted synchronisation of multiple coherent lasers, we demonstrate how it possible to derive phase regeneration in signals with more than two levels of phase encoding.
\end{abstract}

Keywords: All-optical signal processing, optical regeneration, phase sensitive amplification, fiber optical parametric amplification.

\section{INTRODUCTION}

When transmitted over very long distances, the requirement to maintain signal power at a level sufficient to overcome transmission losses means that nonlinear impairments start to be introduced. These degradations normally involve cross or self phase modulation between the single channel data signal, any in-band noise introduced from quantum loss and optical amplification, as well as any neighbouring co-propagating optical data channels. These nonlinear phase impairments can be minimal for short optical links, but rapidly become the major limiting factor for long haul phase encoded optical channels [1]. When phase noise increases to drastic levels, signal regeneration is required to eliminate as much of the accumulated noise as possible prior retransmission. Signal regeneration is currently performed in commercial systems using optical-electrical-optical (O-E-O) conversion. While OEO regeneration, particularly when assisted by forward error correction (FEC), is capable of perfectly restoring the original signal, such regenerators require a full receiver and transmitter per optical channel, implying cost and power penalties, which become more of an issue as symbol rates rise. With this in mind, all-optical solutions remain very attractive. The use of optical nonlinearities in fibers has long been studied for optical regeneration over the last few decades, with demonstrated examples including the Mamyshevregenerator (which employs self-phase modulation in an optical fiber followed by spectral filtering in order to regenerate intensity modulated signals [2]. However, the regeneration of phase-encoded signals is becoming ever more of interest in modern high-speed transmission systems, where the use of such modulation formats is favored for achieving high spectral efficiency. Phase sensitive (PS) optical parametric processes allow direct measurement and manipulation of the optical phase [3], and as such allow all-optical phase regeneration. In the work described here, we demonstrate the regeneration of phase-encoded signals by making use of phasesensitive amplification (PSA) based on parametric processes in highly nonlinear optical fibers (HNLFs). Suitable combinations of such processes allow even the regeneration of signals with multiple levels of phase encoding, by effectively quantizing the phase at the output of the system to a corresponding number of levels [4].

\section{CONCEPT AND EXPERIMENT}

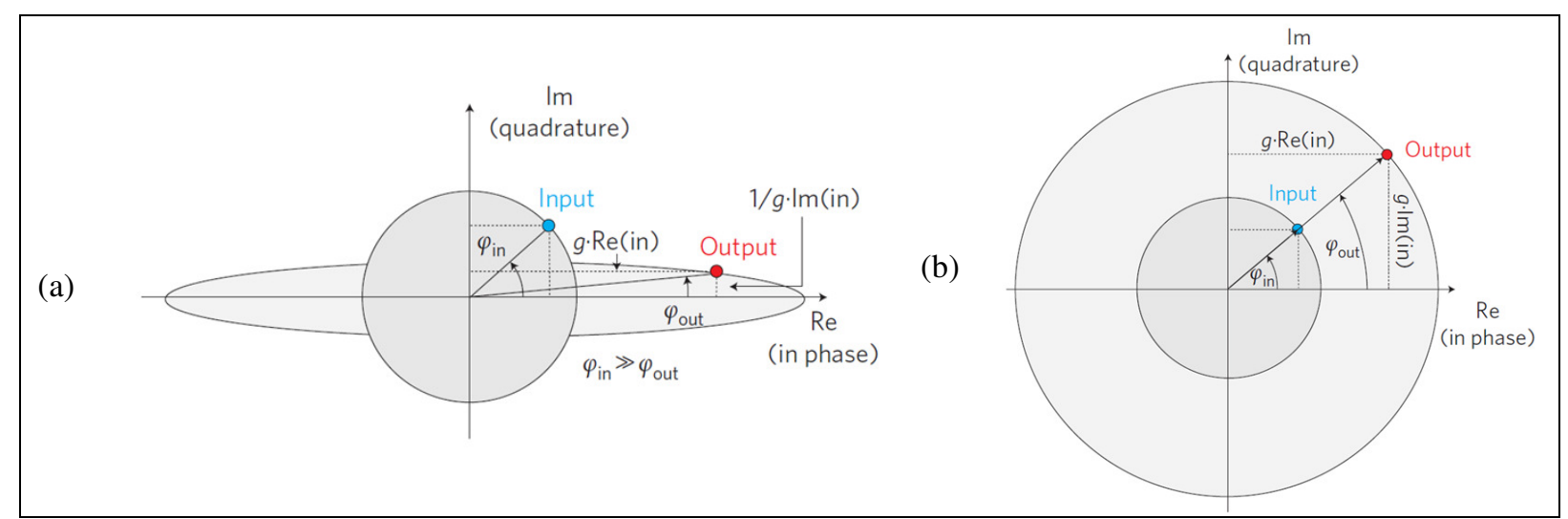

Figure 1: Phase-insensitive (PI) versus phase-sensitive (PS) amplification (taken from [3]). 
Figure 1 highlights the difference between conventional (PI) and phase-sensitive (PS). In a PI amplifier such as an erbium doped fiber amplifier (EDFA), both in-phase and quadrature components of a signal experience identical amplification irrespective of input phase. This PI gain is accompanied by addition to the signal of a certain amount of quantum noise, related to the $3 \mathrm{~dB}$ quantum noise figure of PIs [5]. In a PS amplifier, it is possible to selectively amplify only one of the input signal quadratures while de-amplifying the other. This process allows not only noiseless amplification, but also phase regeneration provided that the quadrature being amplified matches that bearing the data modulation, with the noise confined to the de-amplified quadrature.

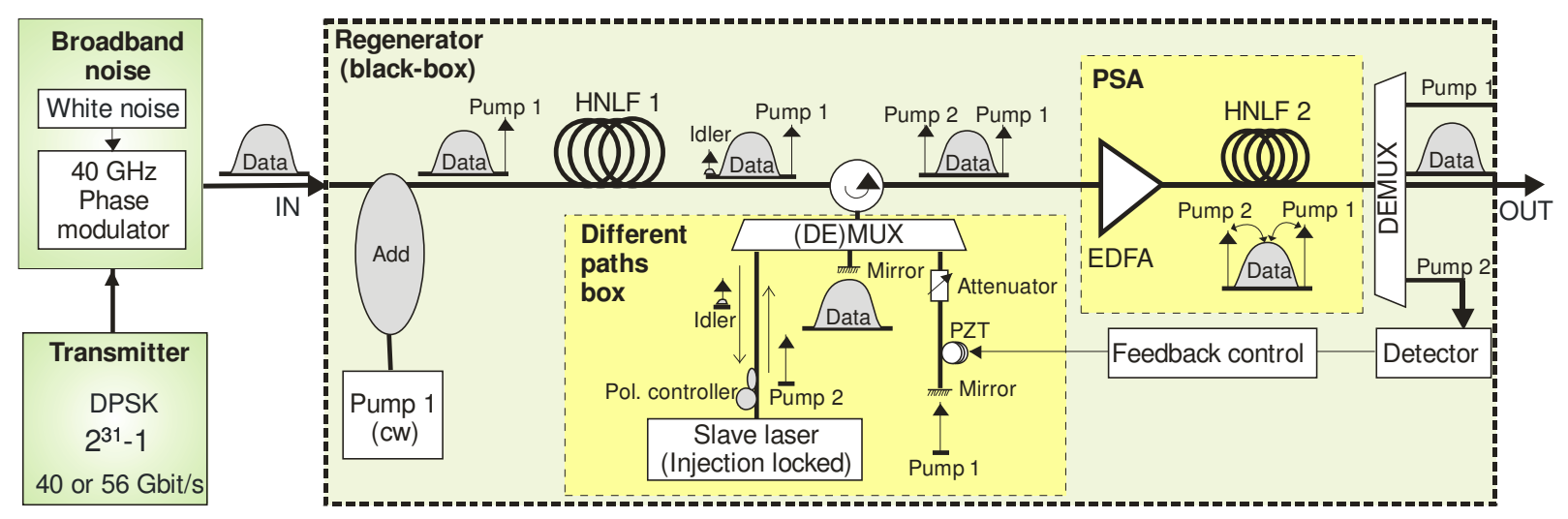

Figure 2: Black-box binary phase regenerator setup. HNLF-Highly Nonlinear Fiber. PZT - Piezo-electric fiber stretcher. EDFA - erbium doped fiber amplifier. DEMUX - optical demultiplexer.

Figure 2 shows the setup for a black box phase regeneration experiment. A $40 \mathrm{Gbit} / \mathrm{s}$ DPSK signal was generated by modulating a Discrete mode semiconductor laser (Eblana Photonics). An electro-optic modulator was used to apply Gaussian phase noise to emulate nonlinear phase noise in transmission links. The signal was combined with a continuous wave (CW) local pump ('Pump 1', its frequency $200 \mathrm{GHz}$ away from the data carrier frequency) using an add-multiplexer giving insertion loss $\sim 0.5 \mathrm{~dB}$. They were then mixed in a polarization maintaining HNLF (OFS Fitel, Denmark). The length, dispersion, nonlinear coefficient and insertion loss of this HNLF were $300 \mathrm{~m}, 1.3 \mathrm{ps} / \mathrm{nm} / \mathrm{km}, 10.5 / \mathrm{W} / \mathrm{km}$ and $0.9 \mathrm{~dB}$ respectively. The semiconductor slave laser was then injection locked to the modulation stripped [3] four wave mixing idler generated in HNLF 1, eliminating all the amplitude noise on the idler, as well as high frequency phase noise. This locally generated beam now serves as pump 2 for the PSA. The data and pumps are launched into the PSA which consisted of a high-power EDFA (total power of $29 \mathrm{dBm}$ ) and a 180-m sample of a high-SBS-threshold HNLF (OFS Fitel, Denmark) [6]. HNLF2 had an alumino-silicate core and had a linear strain gradient along its length. The dispersion, polarization mode dispersion, nonlinear coefficient and attenuation of the fiber were $-0.17 \mathrm{ps} / \mathrm{nm} / \mathrm{km}$, $0.35 \mathrm{ps} / \mathrm{km}^{-0.5}, 7.5 / \mathrm{W} / \mathrm{km}$ and $15 \mathrm{~dB} / \mathrm{km}$, respectively. The relative powers of the pumps and signals were adjusted for optimal regeneration performance (using the attenuator and the bias current of the slave laser in pump paths 1 and 2 respectively). The PZT-fiber stretcher was used as the actuator for a slow feedback loop to compensate for any relative phase drifts picked up due to thermal effects or acoustic pickup experience between HNLF 1 and HNLF 2.
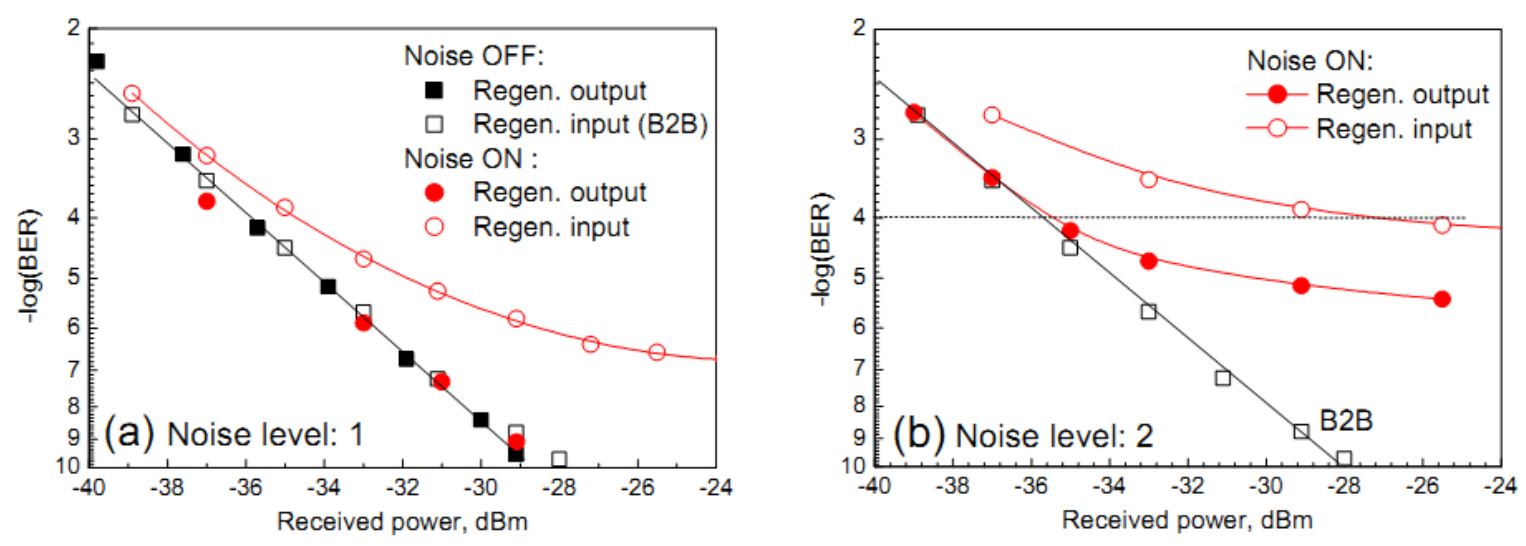

Figure 3: BER curves at the input (open) and output (full) of the regenerator for no added phase noise 
Figure 3 shows the results of bit error ratio (BER) measurements at $40 \mathrm{Gbit} / \mathrm{s}$ at the input and output of the regenerator for various levels of phase noise. In Fig. 3a the level of added noise is such that the regenerator is fully capable of restoring the data fidelity despite the fact that the noisy data exhibited an error floor close to $10^{-7}$ . In Fig. $3 b$ we show the same results for a greater amount of applied phase noise. In this case, the regenerator fully restores the signal fidelity up to BER $10^{-4}$ which is well within the FEC limit. In comparison with the input signal, we obtain a power penalty improvement of $9 \mathrm{~dB}$ at $10^{-4}$. The noticeable BER improvement shown in Fig. 3 is due to a correctable class of errors specific to a 'differential' receiver - e.g., given two consecutive transmitted bits with original phase $0^{\circ}$ which after transmission pick up phase noise such that they are now $+45^{\circ}$ and $-45^{\circ}$ relative to the signal carrier, the demodulated signal after a delay line interferometer would represent a (differential) phase error of $90^{\circ}$ making it impossible to correctly detect the original encoded message clearly resulting in an erroneous detection. This can be fully remedied by the regenerator which acts on the absolute phase of each symbol.

\section{MULTILEVEL PHASE REGENERATION}

Multi-level phase regeneration requires a stair-case phase transfer function. Here, we demonstrate an example with $\mathrm{M}=4$, conventionally known as quadrature phase shift keying (QPSK). QPSK is particularly relevant as it is the modulation format of choice for $100 \mathrm{Gbit} / \mathrm{s}$ Optical Ethernet. We can synthesize an $M$-level phase staircase by coherently adding to the input signal a phase conjugated harmonic (generated by a cascaded FWM process) bearing a temporal phase modulation $M-1$ times that on the signal, analogous to Fourier synthesis of complex signals [4]. The resulting waveform has its phase quantized to $M$-levels and an amplitude response of periodicity $\pi / M$. For QPSK, phase harmonics of the signal are generated using four wave mixing with a CW pump first, Fig. 2 . The $2^{\text {nd }}$ harmonics is subsequently dropped and the $4^{\text {th }}$ harmonic that is CW (as any phase level presented in QPSK of 0, 90, 180, $270 \mathrm{deg}$ multiplied by four gives zero phase level) was used for phase locking of pump 2 in the next stage that was in fact a 2-pump non-degenerate PSA, Fig. 4.

(a)

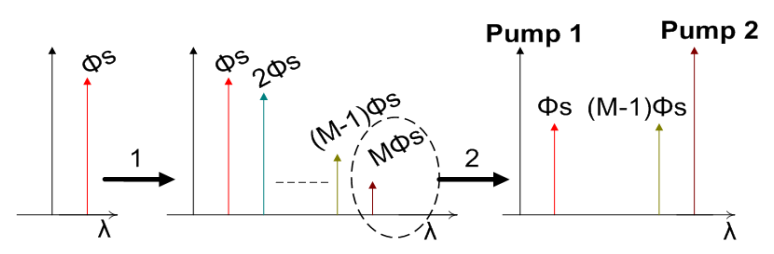

(c)

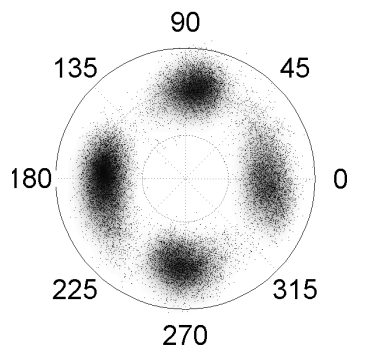

(b)

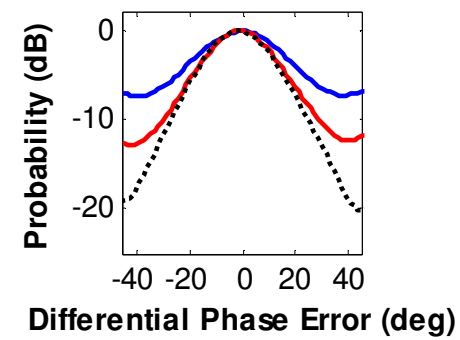

(d)

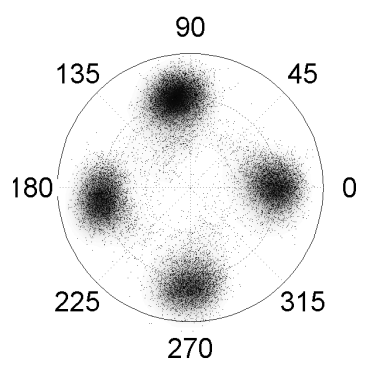

Figure 4: (a) - Regeneration in two steps: (1) Nonlinear generation of phase harmonics FWM followed by pump recovery, (2) Parametric dual pump non-degenerate PSA. (b)- Experimental QPSK differential (symbolto-symbol) phase error distribution, red lines at regenerator output and black dotted line is signal without any added noise. (c), (d) - QPSK input and output constellations respectively.

As an example, a PS mixer operating on this concept was experimentally tested with a 10 Gbaud QPSK input signal. The QPSK signal was degraded using both added Gaussian optical noise and nonlinear phase noise emulated with a phase modulator. Following the PS mixer, the phase and amplitude excursions are significantly reduced. The differential phase error distribution (symbol-to-symbol phase difference) is shown Fig. 4(b). As can be seen, the probability of differential decoded digital errors (symbols with differential error $>45^{\circ}$ ) is 
reduced significantly. The signal constellations before and after the regenerator are also shown in Fig. 4(c) and 4(d) respectively.

\section{CONCLUSIONS}

In conclusion, PSAs now allow for the black-box regeneration of various phase modulation formats by exploiting a new class of optical signal processing functions, advances in highly nonlinear fiber technology as well improved phase locking of high power coherent optical pumps. They can provide significant BER improvements in an asynchronously operated device, providing a degree of flexibility and reconfigurability suited to future transparent optical networks.

\section{ACKNOWLEDGEMENTS}

The authors are grateful to Dr L. Gruner-Nielsen (OFS, Denmark) and Dr R. Phelan (Eblana Photonics, Ireland) for providing respectively the HNLFs and narrow linewidth sources used in these experiments. This research has received funding from the European Communities Seventh Framework Programme FP/2007-2013 under grant agreements 224547 (PHASORS).

\section{REFERENCES}

[1] J. P. Gordon, and L. F. Mollenauer, "Phase noise in photonic communications systems using linear amplifiers," Opt. Lett. 15(23), 1351-1353 (1990).

[2] P. V. Mamyshev, "All-optical data regeneration based on self-phase modulation effect," in $E C O C^{\prime} 98$, Madrid, pp. 475-476, 1998.

[3] R. Slavik et al, "All-optical phase and amplitude regenerator for next-generation telecommunications systems," Nature Photonics, vol. 4, pp. 690-695, 2010.

[4] J. Kakande et al, "First demonstration of all-optical QPSK signal regeneration in a novel multi-format phase sensitive amplifier,” ECOC 2010 Turin 19-23, Italy, PD 3.3, Sep 2010.

[5] C. M. Caves, "Quantum limits on noise in linear amplifiers", Phys. Rev. D 26, 1817 (1982).

[6] L. Grüner-Nielsen et al, "Silica-based highly nonlinear fibers with a high SBS threshold", IEEE Photonics Society 2011 Winter Topical meeting, Paper MD4.2 (2011). 